\title{
Approaching Citizens by City Microblogs: Cases from China
}

\author{
Lijun Zhou \\ Economics School, Kunming University, Kunming, Yunnan, China 650214 \\ junzi588@163.com
}

\begin{abstract}
Keywords: City Microblog, Message Appeal Effect, Wei Chengdu, Shanghai Release, Nanjing Release

Abstract. Advanced internet information technology enables a revolution for people's daily lives. Apart from social network sites, such as Facebook, microblogs have become the second inevitable and significant communication platform all over the world. Common people, celebrities, firms and governmental agencies have been all fascinated to open their own microblogs. In China, a large number of governmental microblogs, especially city microblogs opened by city government agencies have been skyrocketed since 2011. This paper aims at exploring the characteristics which influence over the success of city microblogs in China via a case study approach. Based on the content analysis of city microblog posts from three typical city microblogs in China, this paper suggests the message appeal effect on the success of city microblogs in China.
\end{abstract}

\section{Introduction}

In recent years, as the vigorous development of internet information technology, social media based on the Web 2.0 platform has largely changed people's daily lives. From social network sites (e.g., Facebook), microblogs (e.g., Twitter), video sharing sites (e.g., YouTube) to Wikis (e.g., Wikipedia), these four typical social media categories have enabled common people to share their own materials, or called User Generated Content (UGC) in the virtual reality [1]. Although a microblog is developed relatively later compared with other three categories, it is an inevitable and significant vehicle for people to interactively communicate via computers or mobile devices at any time and any places all over the world.

Since the occurrence of Twitter in 2006, microblog has skyrocketed in popularity all over the world. In China, some local Chinese internet web portals, such as Sina, Sohu and Tencent, have launched the Chinese microblog platforms, called as Weibo which integrates both the functions of Twitter and Facebook since 2008 [2]. A large number of government agencies in China have employed Weibo platform to publish information, guide public opinion and establish government image as well as implement political participation since 2011[2]. As a special type of government microblog, city microblog opened by a city governmental agency aims not only at implementing the functions of normal government microblog, but also at promoting the local culture, city image and serving for the local citizens. Wei Chengdu as a typical city microblog in China, is an official and successful government microblog launched by Chengdu City, the capital city of Sichuan Province located in southwest China. It has become a name card of Chengdu City and the top city microblog in China with more than five million followers since 2012 [3,4]. Furthermore, Shanghai City and Nanjing City also opened their own microblogs named as Shanghai Release and Nanjing Release which has attracted over six and three million fans respectively in 2017. Because of the outstanding performance of city microblogs, like Wei Chengdu, Shanghai Release and Nanjing Release, we would like to address the following questions: what are the characteristics of city microblogs? Why the citizens have engaged actively in their local city microblogs?

\section{From a Microblog to a City Microblog}

A microblog is an exchange instrument which relies on the platform of Web 2.0. It allows users to exchange short contents which include sentences (not more than 140 words), images and video linkages[1]. Because of the exclusive attributes of timeliness, flexibility, participativness, interactivness and openness, the more and more entities, such as individuals and firms begin to 
explore the marketing potential of microblogs [5]. Although microblog marketing is widely used by individuals and enterprises, it also creates a new opportunity for government agencies. Because of the benefits for public participation and instant communication, some governmental agencies utilize this new virtual platform to enhance the relationship between government and the public [5]. Hence, a new microblog type, government microblogs opened by governmental agencies come out for the said purpose. It is a new virtual platform for government agencies to announce information, guide public opinion, promote government image and fulfill public participation [2]. In the US, the birthplace of microblogs, Twitter is an attractive platform for White House to release information for 24 hours a day. It becomes a channel not only to announce messages but also to cultivate public relation via interactive communication between White House and its followers [3].

In China, some local Chinese web portals, such as Sina, Tencent and Sohu, have launched microblog platforms with Chinese characteristics, called Weibo since 2008 [6]. Compared with Twitter, Chinese Weibo platform originally adds the functions of forwarding and reviewing for microblog posts [5]. As a popular social media category utilized in China, microblogs have been largely used for news report and public discussion [6]. In 2017, the number of government microblogs sharply rose over 170,000 based on Sina Weibo platform [7] . Although some microblog accounts are inactive, these striking numbers still implies an impressive power of government microblogs in China [6]. Among these numerous government microblogs, a special group of accounts named by Chinese cities, called city microblogs are very popular in China. City microblogs are launched by municipal departments, such as official news agencies or tourism agencies, not only to implement the basic functions of government microblogs, but also to promote city image and local culture. The main audiences of city microblogs are local citizens [8]. In order to establish a good relationship between a city and its citizens, a city microblog mainly provides public service, such as daily transportation condition, weather information, new house property regulations and other messages related to the citizens' daily lives [5]. It also enables a channel timely to solve problems and receive complaints and suggestions from the citizens via microblog governance $[3,4]$.

Although as a sub-type of government microblog, a city microblog embodies some exclusive attributes with comparison to the other microblogs. Firstly, it is a non-profitable microblog launched by a specific city government agency (e.g., municipal news sector) for information publication and public service. It leverages the name of specific city, such as Wei Chengdu via utilizing the city name of Chengdu City and Shanghai Release by employing the name of Shanghai City. Secondly, posts issued by a city microblog mainly involve all-around information of the citizens' livelihood, such as weather, transportation, housing policies and other messages which refer to the basic necessities of the citizens' daily lives. These messages are typically localized at forming a convenient information sharing platform for the citizens. Thirdly, a city microblog is at promoting the culture and building the good image of a city among the citizens, tourists and investors. Posts on a city microblog not only relate to the local livelihood, but also to promote the local customs, history and other cultural characteristics. A city microblog also initiates some interesting topics, such as personal stories with the city and entertainment gossips related to the city for public discussion. These messages are with high popularity for browsing, reviewing and forwarding.

\section{Research Method}

As current literature on a city microblog is limited, a multiple cases study method can systematically explain the questions of why and how issues related to a city microblog [9]. It also ensures the depth of qualitative research and preferably exploring the background of the cases [9]. Thus, we believe that multiple cases study approach is appropriate to initially explore the secrets of city microblogs. Because of the blowout growth of government microblogs, Wei Chengdu becomes the outstanding player as a typical city microblog to promote the image and culture continuation of Chengdu City rather than only release updating information [3]. Additionally, from the top ten government microblogs of Sina Government Microblog Report in 2016, we selected another two city microblogs, Shanghai Release (i.e., the official microblog of News Office of Shanghai Municipal Government) and Nanjing Release (i.e., the official microblog of Publicity Department of CPC (Communist Party 
of China) Nanjing Municipal Committee) to increase the robustness of our study. Hence, we chose three popular city microblogs as our research cases to explore the successful secrets of city microblogs in China.

\section{Finding: Message Appeal Effect}

A typical characteristic of Wei Chengdu, Shanghai Release and Nanjing Release is equipped with fully emotional expressions in the posts. Especially, some posts related to life feelings and inspirations are very popular among the citizens. For example, Wei Chengdu released a post at 8:01 am, 14 October 2013 in the routine microblog column of "Good Morning, Chengdu" which stated that: "Life cannot always goes well, but continue to walk towards the sun. Then shadow will certainly disappear afterwards. Good Morning, Chengdu, a whole new week is beginning!" This post simulated nearly 960,000 people to browse with 167 reviews and 158 people to forward. Apart from this merely emotional life inspiration post, some factual messages, such as weather forecasting news are also emotional in the posts of the three city microblogs. For instance, Wei Chengdu issued a post on 14 October 2013 which contained not only the weather information (e.g., temperature, windy), but also employed some emotional and humorous sentences to remind people for adding clothes, like "Temperature fall sharply, make sure to wear your long johns. Maybe you need to ransack your wardrobes and cabinets to find them out. Do you wear long johns today?" Nearly 100,000 microblog users read this post which enabled 56 reviews and 55 followers to forward. Moreover, Shanghai Release promotes the night column of "Night reading" which not only recommends a book, but also shares the after-reading comments every day. Sentences like "Do you aspire a free sky?", "Time dilute the fervent love" are presented in the book introduction post which arouse the audiences' thinking emotionally. For Nanjing Release, a routine column of "Nanjing, good night" usually contains the life feeling sentences with specific theme, such as the theme of "the fall in Nanjing" with sentences of "No matter where you are, you should always miss the fall of Nanjing" and the topic of "for yesterday self" with expressions like "What do you want to say for yesterday self?".

Based on the current literature, rational message appeal refers to "objective, factual and verified facts", whereas emotional message appeal is "evaluative, emotional and subjective impressions" [10]. Emotional appeal might be more favorable than rational one for persuasion effectiveness in adverting context [10]. A few scholars also suggested that emotional appeal can lead to strong feelings which strengthen people's memories [11]. However, some researchers proved opposite views that rational message appeal is more effective than emotional appeal[12]. These confused findings show that message appeal (rational VS emotional) can lead to different effect based on diverse context. In our cases, government communication always leads to impressions of high power distance, powerful, rational, and serious. However, in a virtual world (e.g., online context), informal communication breaks the traditional communication approach utilized by a government agency. In microblog circumstance, a city microblog fulfills a platform for a city government to build a "people first" image among the citizens via an emotional and humanized method $[3,13]$. Thus, microblog posts with emotional appeal can stimulate emotional responses from the citizens and decrease the distance between a city government and the citizens. It also represents affinity of government via informal and interactive communication with the citizens via a city microblog. When the citizens perceive the distance narrowing gradually between themselves and the city government, their sense of satisfaction will be increased. Thus, we propose:

Proposition : City microblog posts with emotional appeal are more favorable than posts with only rational facts. They can reduce the distance between a city government and the citizens, and positively affect the citizens' satisfaction of a city microblog.

\section{Conclusions}

As the increased popularity of microblogs all over the world, some Chinese characteristic microblog platforms launched by famous Chinese web portals, such as Tencent and Sina Weibo are very popular in China. Not only for individuals, such as ordinary people and celebrities, but also for organizations, 
such as firms, governmental agencies and social communities, all launched their microblogs to follow the new communication trend. Among these microblogs, a special type of government microblog opened by a city government crowned with the city name, called city microblogs have made an explosive increase in China since 2011 [2]. City governments at different levels launched city microblogs which stimulate many citizens to be the fans of city microblogs. It seems that plenty of usable posts contribute to the popularity of city microblogs. However, are there any other factors which enable the citizens to engage in city microblogs? In order to explore the popularity secrets of city microblogs in China, we conducted a qualitative research via utilizing the cases of Wei Chengdu, Shanghai Release and Nanjing Release, which are the top three city microblogs with average more than four million fans in China. We find that all these three city microblogs are consistent with the basic attributes of microblogs such as visual appeal, entertainment value and customization [14]. However, there is another attribute which Wei Chengdu, Shanghai Release and Nanjing Release exhibit in the issued posts. We find the emotional expressions inserted in the posts issued by Wei Chengdu, Shanghai Release and Nanjing Release. Some factual news posts, such as weather forecasting and traffic condition are inserted with some emotional expressions which demonstrate the concept of "people first" strategy by the city microblogs. These messages are full of emotional appeals which contribute to the popularity of city microblogs via high browsing, forwarding and reviewing rates by audiences.

\section{Acknowledgements}

This work was financially supported by the Scientific Research Foundation of Kunming University, China (No. XJW14007).

\section{References}

[1] A. M. Kaplan and M. Haenlein: Business Horizons, Vol. 54 (2011), p. 105-113.

[2] H. Huang and L.L.Liu: Modern Communication, Vol.3 (2012), p. 122-126 (In Chinese).

[3] J.Liu and M. H. Chen: Press Circles, Vol.9 (2012), p. 30-32 (In Chinese).

[4] L.J. Zhou and T. Wang: Cities, Vol. 37 (2014), p. 27-32.

[5] Y. Hu: The Press, Vol. 9 (2011), p. 17-20 (In Chinese).

[6] B.L.Cao:Journal of Hunan Radioand Television University, Vol. 47(2011), p. 65-69 (In Chinese).

[7] Information on http://www.southen.com/.

[8] Y. Hu, Y. J. Liu and Y. G. Cai: Science and Technology for China's Mass Media, Vol.9 (2012), p. 55-58 (In Chinese).

[9] R.K. Yin: Case Study: Design and Method, (Chongqing University Press, China 2004).

[10] M. B. Holbrook: Journal of Marketing Research, Vol.15 (1978), p. 545-566.

[11] T. J.Page, E. Thorson and M. P. Heide, in: Emotion in Advertising, edtied by S.J. Agres, J.A. Edell and T.M. Dubitsky, Quorum Books, New York (1978), p. 255-268.

[12] L.L.Golden and K.A. Johnson: Advances in Consumer Research, Vol.10 (1983), p. 203-208.

[13] T. Zheng: Library and Information Service, Vol.3 (2012), p. 23-28 (In Chinese).

[14] L.C.Harris and M.M.H. Goode: Journal of Services Marketing, Vol.24 (2010), p. 230-43. 